\title{
Osteoma trabecular: relato de caso
}

\author{
Osteoma trabecular: relato de caso \\ Trabecular osteoma: report of case \\ Vivian Bentes de OLIVEIRA \\ Analu Vieira do NASCIMENTO \\ Lioney Nobre CABRAL \\ Tiago Novaes PINHEIRO \\ Myrian Salles VIEIRA \\ Antônio Jorge Araújo de VASCONCELOS II
}

Escola Superior de Ciências do Amazonas - Universidade do Estado do Amazonas, ESA-UEA, 69050-030 Manaus - AM, Brasil

\section{Resumo}

Introdução: O osteoma é um tumor benigno, de crescimento lento e indolor, composto de osso maduro que pode ser compacto e/ou esponjoso, afetando geralmente os ossos gnáticos. Objetivo: mostrar um caso clínico de osteoma trabecular, que afetou região edêntula do elemento 36 em paciente do sexo feminino, feoderma de 36 anos onde, há aproximadamente 3 anos, o elemento fora extraído devido à destruição por cárie e não feito nenhum tratamento reabilitador. Material e método: Realizado exame clínico e tomográfico, sendo neste observado em corpo mandibular, na região edêntula do elemento 36 , uma imagem hiperdensa irregular, mas bem delimitada de aproximadamente $5 \mathrm{~cm}$ em seu maior diâmetro, circunscrita por área hipodensa. A interrelação desses exames levou às hipóteses diagnósticas de osteoma, osteoblastoma e odontoma complexo, que, após biópsia excisional e análise histopatológica, se confirmou o diagnóstico de osteoma. Resultado: Realizada a interrelação clínica, imagenológica e histopatológica, obteve-se o diagnóstico de osteoma trabecular. Conclusão: a remoção completa da lesão foi importante para a confirmação diagnóstica e configurou seu tratamento, sendo raros os casos recidivantes.

Descritores: Osteoma; Neoplasias Bucais; Cirurgia Bucal.

\section{Abstract}

Introduction: Osteoma is a benign neoplasm, slow growing, and usually asymptomatic, composed of mature bone which can be dense and/or cancellous often affecting the gnathic bones. Objective: Report a case of trabecular osteoma which occurred in lower left first molar edentulous region of female patient, feoderma, 36-years-old. About 3 years ago tooth was extracted because of caries and no rehabilitation treatment was performed. Materials and methods: after clinical and tomographic exam it was observed in edentulous region in body of mandible a hyperdense image, well circumscribed, measuring about $5 \mathrm{~cm}$ in its greatest dimension, surrounded by hypodense area. The correlation of all the exams conducts to osteoma, osteoblastoma and complex odontome as diagnostic hypotheses. Excisional biopsy and histopathological analysis confirmed diagnosis of osteoma. Results: correlating clinical, imagenological and histopathologic findings a final diagnosis of osteoma trabecular. Conclusion: complete lesion excision was important for diagnostic clarification and represented its treatment, being rare recurrences.

Descriptors: Osteoma; Mouth Neoplasms; Surgery, Oral.

\section{Resumen}

Introducción: El osteoma es un tumor benigno, de crecimiento lento y indolor, compuesto de hueso maduro que puede ser compacto o esponjoso, que afecto la región sin diente del elemento 36 en paciente del sexo feminino, feoderma de 36 años donde aproximadamente hace 3 años, el elemento fue retirado debido a la destrucción por cárie y haber realizado un tratamiento rehabilitado. Material y método: realizado examen clínico y tomografico, siendo en este observado en cuerpo mandibular, en la región sin diente del elemento 36, una imagen hiperdensa irregular, pero bien delimitada de aproximadamente $5 \mathrm{~cm}$ en su mayor diámetro, circunscrita por área hipodens, la interrelación de los exámenes llevó a hipótesis diagnóstica de osteoma, osteoblastoma y odontoma complejo, que después de la biopsia escisiónal y análisis histopatológicas, se confirmó el diagnóstico de osteoma trabecular: Conclusión: remoción completa de la área afectada, fue importante para la confirmación diagnóstica y después configuro su tratamiento, siendo raros los casos de recaída.

Descriptores: Osteoma; Neoplasias de la Boca; Cirugía Bucal.

INTRODUÇÃO

O osteoma é um tumor benigno, de crescimento lento e indolor, composto de osso maduro que pode ser compacto e/ou esponjoso afetando comumente ossos gnáticos. É classificado de acordo com sua localização, quando surge na periferia óssea é chamado de periosteal (periférico ou exofítico), quando se origina em osso medular, denomina-se endosteal ou central. Geralmente é diagnosticado quando há assimetria facial e/ou interferência direta na função normal de sua localização anatômica. Sua etiologia ainda é desconhecida, mas pode estar associada a traumas, infecções e anormalidades do desenvolvimento. As áreas mais acometidas são o corpo da mandíbula ou o côndilo ${ }^{1,2}$. A faixa etária mais acometida é entre 20 e 50 anos. Na maioria das vezes é solitário, quando há múltiplos osteomas, podem estar associados a Síndrome de Gardner ${ }^{3-5}$. O osteoma periférico se apresenta como aumento de volume ósseo séssil ou pedicular, já o central geralmente é encontrado em exames radiográficos de rotina, ou quando há aumento de volume da área acometida, levando a assimetria ${ }^{5}$.

O diagnóstico de osteoma é estabelecido com o auxílio das informações clínicas combinadas a recursos imageneológicos e análise das características histológicas, estas informações são importantes para descartar hipóteses diagnósticas de osteoblastoma e odontoma complexo ${ }^{6}$. O osteoblastoma quando afeta os ossos gnáticos (raramente), acomete a região de mandíbula, região posterior, na maioria dos casos e está associado a dor, sensibilidade e aumento de volume $e^{5,7}$. O odontoma complexo é assintomático, com maior incidência na mandíbula, sendo geralmente 
diagnosticado em exames radiográficos de rotina, ou quando a queixa é o atraso na erupção de um dente, são lesões relativamente pequenas, mas que podem tomar grandes proporções $^{5,8}$.

Em exame radiográfico, o osteoma cortical mostra padrão esclerótico uniforme ou com uma periferia esclerótica com padrão trabecular central, sendo mais fácil o diagnóstico por estar na superfície óssea, enquanto o osteoma central se exibe como massa esclerótica circunscrita no interior do osso, envolta por cápsula de aparência radiolúcida, que em seu início de desenvolvimento pode se assemelhar a focos de osso esclerótico representando o estágio final de um processo inflamatório ou nãoinflamatório ${ }^{3,5}$. O osteoblastoma, pode se apresentar como uma lesão bem definida, ou não, com consideráveis áreas de mineralização $0^{5,7}$. O odontoma aparece como uma massa calcificada, onde a sua densidade é similar a da estrutura dentária, estando cercada por uma delgada margem radiolúcida, podendo ser confundido com osteoma ${ }^{5,8}$.

A tomografia computadorizada é um exame complementar importante no diagnóstico de patologias, em especial patologias ósseas, assim como no planejamento cirúrgico e acompanhamento destas lesões, pois apresentam resolução e precisão das imagens obtidas. No osteoma, o padrão periosteal se apresenta como uma massa óssea com envolvimento da cortical externa do osso, já o central mostra uma massa óssea hiperdensa bem delimitada no interior do osso que pode estar envolta por imagem hipodensa. Quando a exérese cirúrgica está indicada, a tomografia auxilia no seu planejamento, pois dá precisão quanto à localização, tamanho e forma do osteoma $^{6,8}$.

$\mathrm{Na}$ microscopia, o osteoma apresenta tecido ósseo maduro, podendo ser compacto (cortical) ou medular (esponjoso ou trabecular), no primeiro há presença de osso denso de aparência normal, mostrando tecido medular mínimo e no segundo há trabéculas ósseas, medula fibrogordurosa abundante e a atividade osteoblástica pode estar levemente aumentada $^{3,5}$. O osteoblastoma se apresenta como material mineralizado com linhas reversas proeminentes e por vezes grandes lençóis de trabéculas irregulares ${ }^{5,7}$. Os odontomas complexos em sua maior parte, apresentam dentina tubular madura, esta apresenta fendas, causadas pela remoção do esmalte durante a descalcificação, pode apresentar pequenas ilhas de células epiteliais eosinofílicas e em sua periferia, pode haver uma fina camada de cemento $^{5,8}$.

O tratamento do osteoma pode ser conservador ou cirúrgico. Em osteomas pequenos, assintomáticos e sem qualquer queixa do paciente, pode-se optar por tratamento conservador com acompanhamento clínico e radiográfico, não sendo realizada intervenção cirúrgica, sendo raros os casos de malignização. Nas condições sintomáticas, com alterações funcionais ou deformidades estéticas, ou para fins diagnósticos, o tratamento é cirúrgico, onde há remoção de todo o tumor, seguido de osteoplastia. Quando feita a remoção completa da lesão, são raros os casos de recidiva ${ }^{10}$. No osteoblastoma o tratamento é cirúrgico, com remoção completa da lesão e curetagem, há rara recidiva e malignização ${ }^{5,7}$. O odontoma, geralmente tem como tratamento excisão local simples com ótimo prognóstico ${ }^{5}$.

CASO CLÍNICO

Paciente do sexo feminino, 36 anos, feoderma, procurou Clínica de Estomatologia da Escola Superior de Ciências do Amazonas Universidade do Estado do Amazonas, ESAUEA, Manaus-AM, Brasil, após ser identificada lesão radiopaca em região edêntula em hemimandíbula esquerda, relacionada ao elemento 36 removido, com queixa de tumefação gengival endurecida na região, sem associação a qualquer sintomatologia dolorosa. O elemento 36 fora extraído há aproximadamente 3 anos, devido à destruição por cárie, não sendo realizado nenhum tipo de tratamento reabilitador. Quanto à história médica e familiar não relatou nada digno de nota. Foi realizada tomografia computadorizada para investigação, visualização do aspecto e abrangência da lesão, bem como busca de outros sítios com características similares, não sendo encontrados. No interior do corpo mandibular em região edêntula do elemento 36 , foi observado uma imagem hiperdensa irregular, mas bem delimitada de aproximadamente $5 \mathrm{~cm}$ em seu maior diâmetro, circunscrita por área hipodensa (Figuras 1 a 4) e em vista 3D encontrada uma elevação, mas sem projeção, da área quando comparada ao nível ósseo dos elementos dentários vizinhos (Figura 5). Após associação de informações clínicas e imagenológicas surgiram hipóteses diagnósticas que compreendiam osteoma, osteoblastoma e odontoma. Foram dadas a paciente as opções de não intervir cirurgicamente e ter acompanhamento radiográfico para acompanhar se haveria crescimento ou intervenção cirúrgica através de biópsia 
excisional com acompanhamento radiográfico para analisar se haveria recidiva e obter 0 diagnóstico definitivo da lesão, a mesma optou por intervenção cirúrgica com acompanhamento radiográfico. Foram então solicitados os exames laboratoriais: hemograma completo, coagulograma e glicemia em jejum, para investigação de alguma doença de base ou alteração sistêmica que impossibilitassem a realização do procedimento cirúrgico, os resultados estavam dentro da normalidade. No dia da cirurgia foi realizada a aferição da pressão sistólica, diastólica e frequência cardíaca, estas dentro do padrão de normalidade.

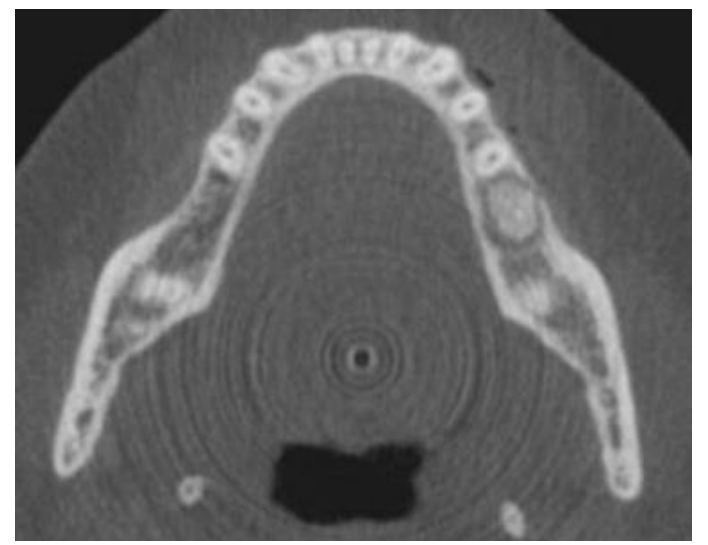

Figura 1: Tomografia Computadorizada (corte axial): visão geral da mandíbula que evidencia imagem hiperdensa de formato arredondado envolta por área hipodensa bem delimitada (azul), limites definidos e corticalizados na região edêntula do elemento 36 .

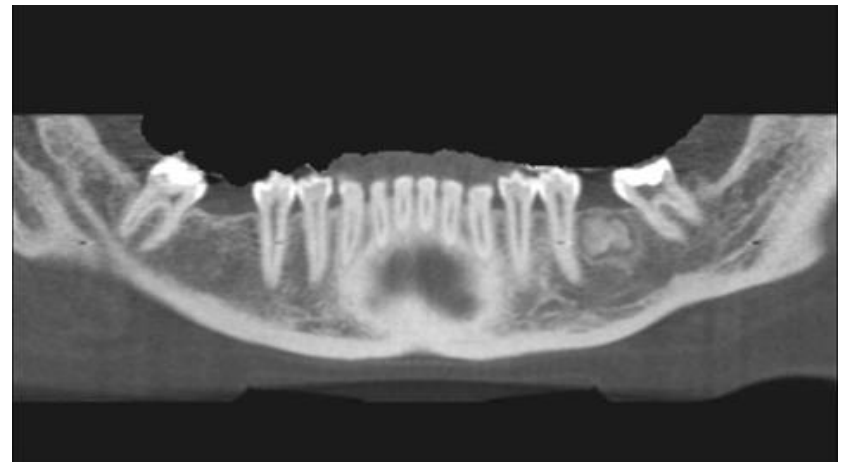

Figura 2: Tomografia Computadorizada (corte coronal): imagem hiperdensa irregular, mas bem delimitada envolta por área hipodensa bem delimitada em região edêntula do elemento 36 .

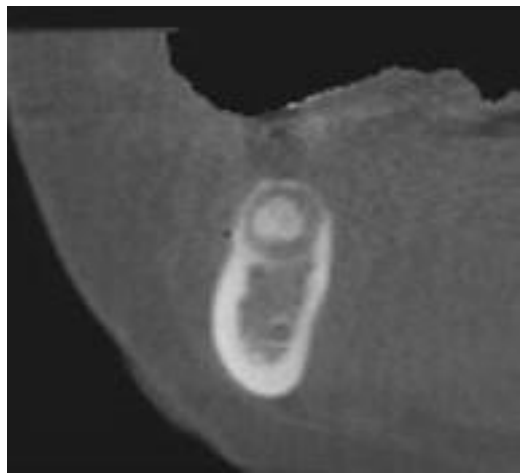

Figura 3: Tomografia computadorizada (corte transversal): imagem hiperdensa de formato arredondado envolta por área hipodensa bem delimitada, limites definidos e corticalizados no interior do corpo mandibular da região edêntula do elemento 36 .

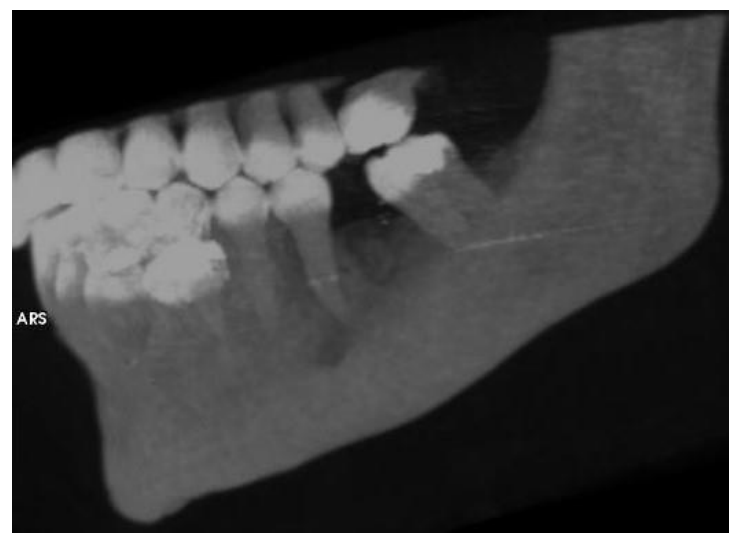

Figura 4: Tomografia Computadorizada - Corte Sagital: imagem isodensa envolta por área hipodensa em região edêntula do elemento 36.

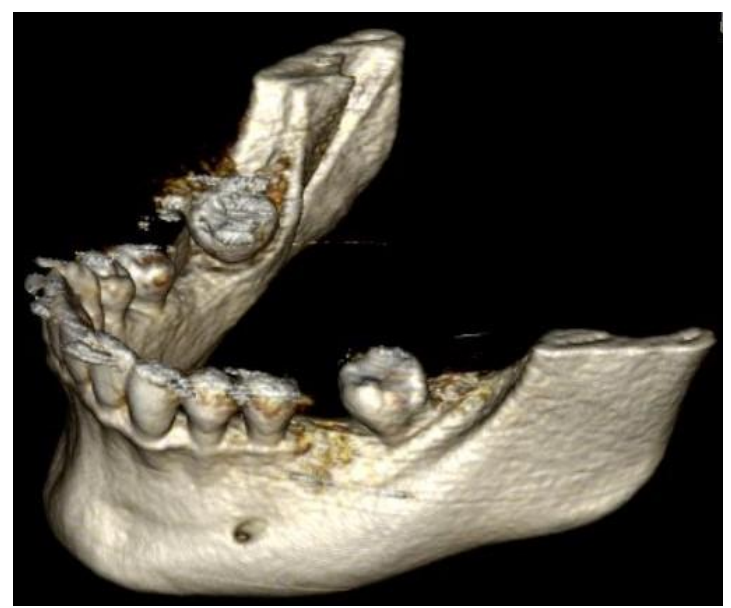

Figura 5: Tomografia Computadorizada - Vista lateral esquerda em 3D: elevação óssea, sem projeção tendo como comparativo o nível ósseo dos elementos dentários vizinhos.

Foi realizado exame clínico intraoral mais uma vez para análise da região que passaria por intervenção cirúrgica, a mesma encontrava-se com as mesmas características das vistas no exame inicial (Figura 6).

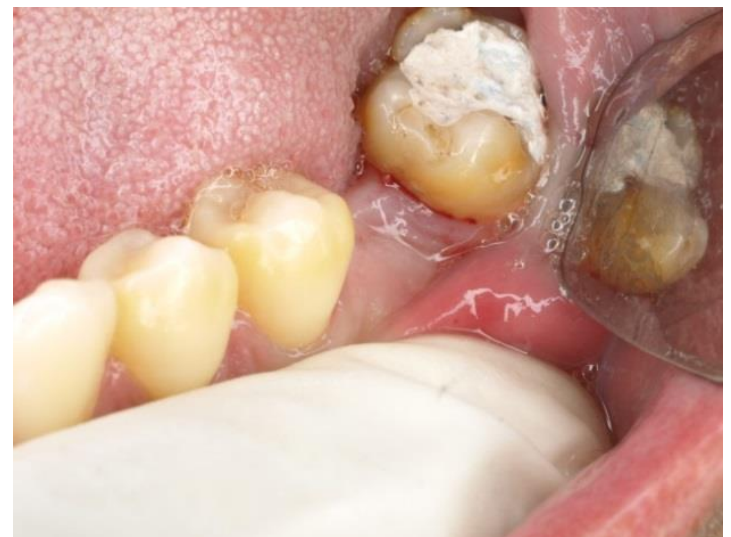

Figura 6: Mucosa se apresenta integra e saudável, tendo leve tumefação.

Foi feita antissepsia intra e extraoral, seguida de técnica anestésica utilizando Articaína 4\% com epinefrina1:1000 associado a Lidocaína $2 \%$ com epinefrina 1:100.000, sendo feito o bloqueio do nervo alveolar inferior, lingual e bucal. Foi feita incisão de Newman modificada com lâmina $15 \mathrm{c}$ em região edêntula do elemento 36 se estendendo levemente para o 
elemento 35 e 37 . Foi utilizada caneta de alta rotação com irrigação e broca carbide esférica cirúrgica $\mathrm{n} \div 4$, sendo removido fragmento de consistência dura, forma e superfície irregulares, coloração esbranquiçada, com dimensões de $1,0 \times 0,8 \times 0,3 \mathrm{~cm}$ (Figura 7), seguida de regularização óssea da região (Figura 8).

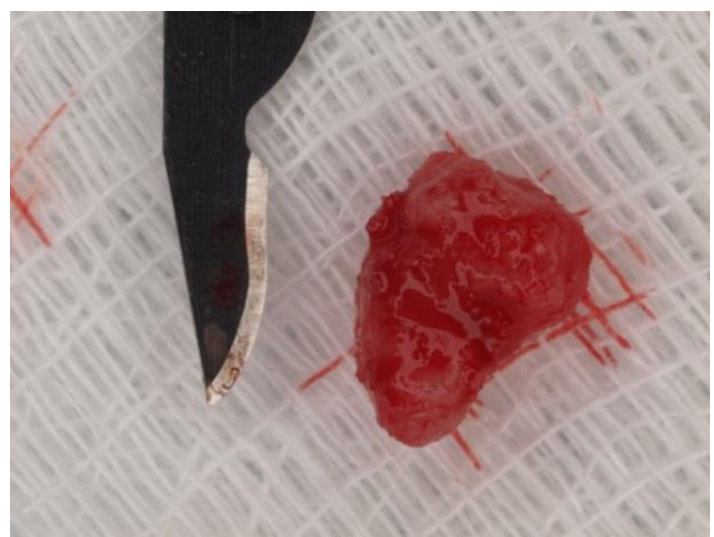

Figura 7: Fragmento de consistência dura, forma e superfície irregulares, coloração esbranquiçada, com dimensões de $1,0 \times 0,8 \times 0,3 \mathrm{~cm}$.

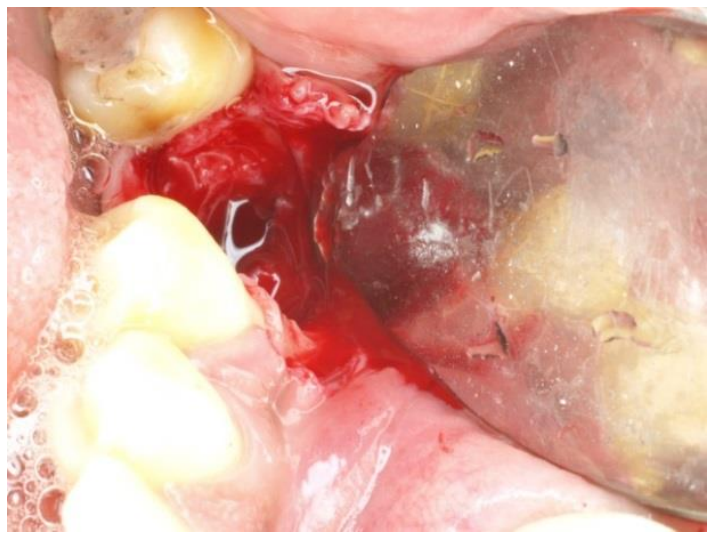

Figura 8: Área após remoção da lesão e regularização óssea, evidenciando incisão do tipo Newman modificada.

Foi realizada sutura com 2 pontos simples utilizando fio de seda (Figura 9). Após a cirurgia, foi prescrita medicação anti-inflamatória por dois dias e analgésico, foram feitas as recomendações pós-operatórias.

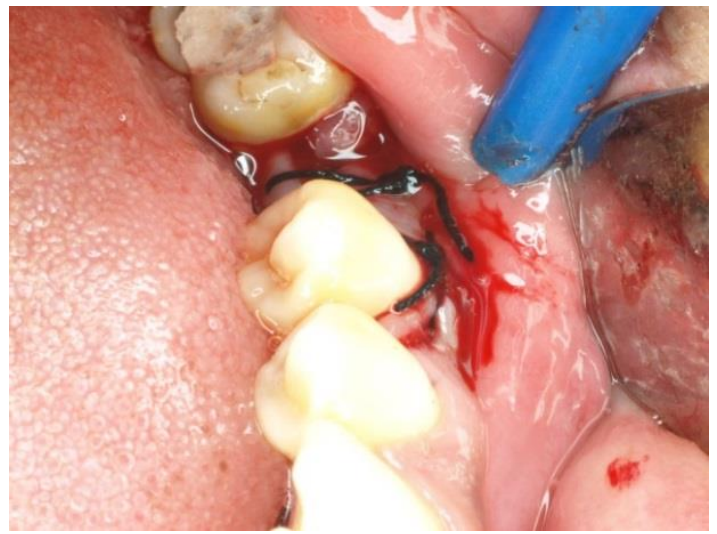

Figura 9: Área após sutura com 2 pontos simples utilizando fio de seda

O material coletado foi encaminhado ao Serviço de Patologia Oral e Maxilofacial da
Instituição dos autores, onde foi submetido à análise histopatológica. Os cortes microscópicos apresentavam cápsula de tecido conjuntivo frouxo não modelado (Figura 10) e organização trabecular com tecido conjuntivo frouxo no interior dos espaços medulares (Figura 11), tendo como diagnóstico osteoma trabecular. A paciente não apresentou recidiva da lesão. Ao final do tratamento, paciente foi encaminhada para reabilitação odontológica.

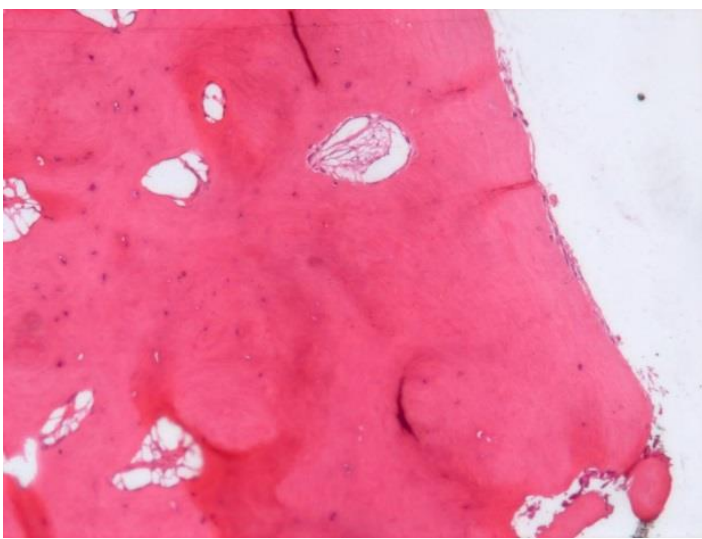

Figura 10: Cápsula de tecido conjuntivo frouxo não modelado.

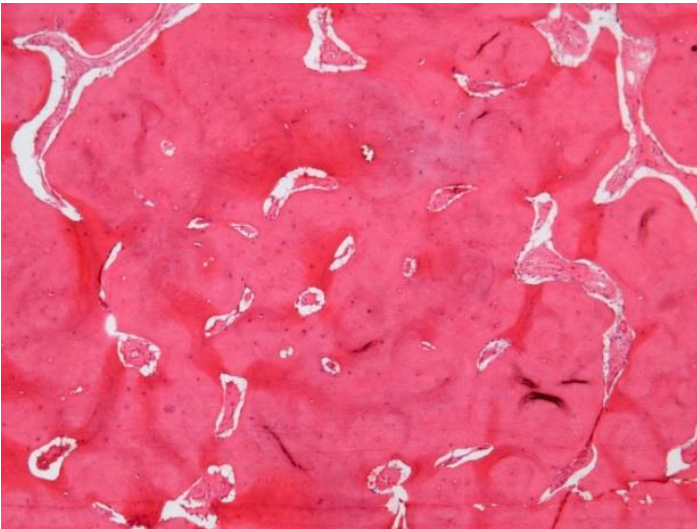

Figura 11: Organização trabecular com tecido conjuntivo frouxo no interior dos espaços medulares.

DISCUSSÃO

O osteoma é uma neoplasia osteogênica benigna, que consiste em osso bem diferenciado e maduro, sendo compacto e ou esponjoso ${ }^{10}$. O sexo feminino e a segunda década de vida mostram mais acometimento ${ }^{11}$. Afetou paciente do sexo feminino, sendo o gênero mais acometido, idade de 36 anos, esta não é a década mais afetada.

A etiologia do osteoma ainda não está clara, embora algumas hipóteses indiquem relação com trauma, processos inflamatórios, hereditariedade, alteração do metabolismo do cálcio e também alterações relacionadas à sobrecarga muscular, porém nenhuma delas foi confirmada $^{12}$. A principal suspeita é que o tumor possa ter se desenvolvido por conta de um processo infeccioso crônico causado por cárie e pelo trauma da exodontia do elemento 36.

Os osteomas não são agressivos e 
geralmente não estão associados à sintomatologia ${ }^{13}$. Assim, muitos casos são diagnosticados de forma incidental, comumente a partir de exames radiográficos de rotina. Para fazer o diagnóstico de osteoma, deve haver evidência de crescimento, expansão ou deslocamento dos dentes ${ }^{14}$. $\mathrm{O}$ caso mostra que paciente não tinha nenhum evento disestésico relacionado à lesão, bem como aumento de volume ósseo exagerado que levasse ao afastamento dos elementos dentários próximos, bem como a assimetria facial, a lesão só foi notada por conta de um achado radiográfico e percepção da paciente quanto à tumefação na área.

Os osteomas podem ser periféricos ou centrais, cerca de 93,4\% são periféricos e apenas $6,6 \%$ centrais, uma proporção de $14: 1^{15,16}$. O osteoma periférico afeta comumente a região de côndilo mandibular e o central também acomete a mandíbula, principalmente região de pré-molares e molares de ambos os lados. No exame de imagem foi notado que a massa tumoral se encontrava intraóssea (osso medular/trabecular) na mandíbula e não em sua superfície (cortical), sendo considerado um evento pouco comum, a região acometida foi a de molar (elemento 36), sendo a mandíbula a mais afetada tanto em osteomas periféricos quanto centrais ${ }^{6,14,16}$.

O tecido ósseo cortical e o tecido ósseo esponjoso possuem os mesmos elementos constitutivos quanto a células e matriz óssea tendo, no entanto, importantes diferenças estruturais e funcionais. O tecido ósseo cortical desempenha fundamentalmente funções de suporte e proteção, estando localizado externamente em relação ao tecido ósseo trabecular, que constitui o eixo da função metabólica e maior atividade de remodelação e, assim tendo, uma resposta mais rápida (para o bem e para o mal) aos estímulos mecânicos, químicos e hormonais, desta forma a velocidade da remodelação do osso esponjoso ou trabecular é muito maior que a do osso cortical $^{10,17}$. O padrão ósseo encontrado na microscopia revelavam uma organização de tecido ósseo trabecular com tecido conjuntivo frouxo no interior dos espaços medulares, isso pode ter relação a velocidade e remodelação do tecido ósseo trabecular, pois ocorre de forma mais rápida.

Radiograficamente os osteomas se apresentam como massas radiopacas com bordas bem definidas e compartilham das mesmas características histopatológicas, onde há a presença de osso maduro compacto e ou trabecular $^{6,11,12}$. As lesões radiopacas centrais dos maxilares, no entanto, requerem uma combinação de investigação clínica, radiográfica e histológica para que seja feito um diagnóstico definitivo. A histologia por si só é insuficiente, pois várias lesões têm características semelhantes e são constituídas por osso maduro de densidades variadas. A histologia pode, no entanto, ajudar a descartar outras hipóteses diagnósticas ${ }^{15}$. Quando feitas as inter-relações clínicas, imagenológicas do caso, as hipóteses quanto ao diagnóstico da lesão abrangiam osteoma, osteoblastoma e odontoma, para elucidação foi realizada biópsia excisional e o material coletado foi submetido à análise histopatológica.

O osteoblastoma, quando ocorre nos maxilares, tem predileção pela mandíbula, afeta mais o sexo masculino na $2^{\underline{a}}$ e $3^{\text {a }}$ década de vida, geralmente crescem lentamente com sintomatologia mínima, mas lesões agressivas podem causar sintomas graves, como edema de partes moles e rigidez articular. A dor é o sintoma de apresentação mais comum e o diagnóstico pode demorar até dois anos a partir do início dos sintomas ${ }^{15,18}$. Sua aparência em técnicas de imagem geralmente mostram os osteoblastomas como tumores osteolíticos; no entanto, devido a componentes osteóides e ósseos, pode ser observada mineralização do tipo ósseo. As lesões geralmente são bem circunscritas, centrais a ligeiramente excêntricas em ossos longos, arredondadas ou ovóides e expansíveis. Histopatológicamente exibem trabéculas ósseas irregulares, com rica vascularização e eritrócitos extravasados ${ }^{15,19}$. Embora 0 achado radiográfico apresentasse semelhança com a que ocorre no osteoblastoma, a história clínica não condizia com que a ocorre na maioria dos casos e se descartou tal hipótese após análise histopatológica.

O odontoma complexo é um hamartoma classificado como tumor odontogênico misto, assintomático, ocorrendo com muita frequência nas primeiras duas décadas de vida, sexo feminino e região de molares de ambos os maxilares. Em exame de imagem, apresenta lesão óssea hiperdensa com halo radiolucido e em sua histopatologia, dentina tubular madura, com fendas após a descalcificação do esmalte e pequenas ilhas de células fantasmas epiteliais eosenofílicas. No caso em questão, embora não tenha sintomatologia clínica dolorosa e o exame radiográfico seja similar a de um odontoma complexo, a histopatologia descartou tal hipótese ${ }^{20}$.

O diagnóstico do caso foi de osteoma trabecular, este afetando região intraóssea 
mandibular. Após o diagnóstico, descartou-se relação com a síndrome Gardner, pois não foi encontrado na tomografia computadorizada outro sítio similar a lesão. O osteoma faz parte de um dos sinais da tríade da síndrome de Gardner, que engloba também polipose adenomatosa dos cólons e cistos epidermóides ${ }^{16,21,22}$.

Osteomas pequenos geralmente não influenciam na estética, nem na função e pelo fato de não apresentar malignização e recorrência, uma abordagem conservadora pode ser realizada com 0 devido acompanhamento pós-operatório, logo a intervenção cirúrgica se dá quando há queixa estética e ou funcional. A cirurgia é o tratamento para esses casos, concorrendo para a cura, sem possibilidade de recidiva, quando da remoção completa da lesão, sendo o diagnóstico final confirmado pela histopatologia. Apesar de serem lesões benignas, deve-se dar ênfase ao planejar sua remoção para ocorra a remoção total do tumor. A reconstrução, quando necessária, pode levar enxertos ósseos livres ou vascularizados, aumentando as chances de um bom resultado morfológico e funcional ${ }^{24}$. O tratamento visa o restabelecimento estético e funcional $^{23,34}$. O tumor ósseo encontrado é pequeno, no entanto para elucidação diagnóstica foi necessário 0 procedimento cirúrgico, também devido ao tamanho do tumor, optou-se pela remoção completa da lesão, o que se tornou o seu tratamento.

CONCLUSÃO

Os osteomas são tumores benignos raros, existem osteomas corticais e centrais, estes se encontram escassos na literatura. Apesar de não haver etiologia comprovada ainda, supõe-se que o caso em questão possa estar associado ao potencial contexto infeccioso crônico e também traumático envolvendo 0 elemento 36. É importante a interrelação clínica com exames imagenológicos e histopatológicos para chegar à conclusão diagnóstica, pois o osteoma central pode ser confundido com outras condições. A biópsia excisional do tumor configura também como seu tratamento, sendo raros os casos de recidiva, como no presente caso.

\section{REFERÊNCIAS}

1. Silva WCN, Araújo BL, Figueiredo EL, Vasconcelos RJH, Bortoli MM. Tratamento cirúrgico de osteoma em mandíbula. Arch Health Invest. 2018:7 (Spec Iss 1):57.

2. Reis Neto RD, Assis MR, Monteiro NG, Silva $\mathrm{JR}$, Mandarino S, Ferreira PHSG et al. Utilização de osteoplastia para tratamento cirúrgico de osteoma. Arch Health Invest. 2018:7 (Spec Iss 3):477.

3. Regezi JA, Ciubba JJ, Morgan RK. Patologia bucal: correlações clinicopatológicas. $5^{\mathrm{a}}$ ed. Rio de Janeiro: Elsevier; 2008.

4. Gumusok M, Degerli S, Toprak ME, Seckin A, Kaya E, Senguven B. Peripheral osteoma of the mandible: a case report. J Istanb Univ Fac Dent. 2015;49(1):47-50.

5. Neville BW, Allen CM, Damm DD, Allen CM, Jerry E. Patologia: Oral \& Maxilofacial. 3‥ ed. Rio de Janeiro: Elsevier; 2009.

6. Tenório JR, Silva TJ, Oliveira DH, Queiroz LM. Osteoblastoma in the retromolar region: a case report. RGO Rev Gaúch Odontol. 2016; 64(2):203-6.

7. Magnabosco Neto AE, Capella DL. Tratamento conservador de grande odontoma complexo em mandíbula. RFO UPF. 2011;16(3):317-21.

8. Rebouças DS, Ferreira TG, Andrade EL, Fortuna TC, Assis AF, Freitas CE. Abordagem cirúrgica de osteoma em osso frontal: relato de caso. Rev cir traumatol buco-maxilo-fac. 2014;14(3):9-14.

9. Khandelwal P, Dhupar V, Akkara F. Osteoma periférico incomumente grande da mandíbula Um caso raro. J Clin Diagn Res. 2016;10(11):ZD11-12.

10. Carvalho RW, Antunes AA, Melo MR, Andrade ES, Pereira CU. Osteoma craniofacial: estudo de 35 casos. Rev Bras Cir Cabeça Pescoço. 2008;37(4):212-14.

11. Larrea-Oyarbide N, Valmaseda-Castellón E, Berini-Aytés L, Gay-Escoda C. Osteomas of the craniofacial region. Review of 106 cases. J Oral Pathol Med. 2008;37(1):38-42.

12. Mafra RP, Vasconcelos RG, Queiroz LMG, Vasconcelos MG. Osteoma maxilar: relato de caso. RBCS. 2012;18(1):49-54.

13. Kaplan I, Nicolaou Z, Hatuel D, Calderon S. Solitary central osteoma of the jaws: a diagnostic dilemma. Oral Surg Oral Med Oral Pathol Oral Radiol Endod. 2008;106(3):e22-9.

14. Castro JF. Oncologia Oral. Recife: Ed Univ UFPE. Capítulo 10; Tumores benignos Osteoma. 2005. p. 152-153.

15. Agnollitto PM, Barbosa MH. Qual o seu diagnóstico? Radiol Bras. 2012; 45(4):XI-XII.

16. Cesa TS, Giustina JCD, Silva AF, Disenha JL, Sassi LM. Osteoma de mandíbula: relato de caso. Rev cir traumatol buco-maxilo-fac. 2013;13(1):59-64.

17. Judas $F$, Palma $P$, Falacho RI, Figueiredo $\mathrm{H}$. Estrutura e dinâmica do tecido ósseo. Texto de apoio para os alunos do Mestrado Integrado em Medicina Disciplina de Ortopedia, 2012.

18. Limaiem F, Byerly DW, Singh R. Osteoblastoma. In: StatPearls [Internet]. Treasure Island (FL): StatPearls Publishing; 2020. 
19. Physiopedia contributors. Osteoblastoma [Internet]. Physiopedia. Jul 201.

20. Thompson LD, Bishop JA. Head and neck pathology. Elservie; Chapter 15 Benign neoplasm of the gnathic bone; 2019. p.414-16.

21. Cruz IA, Vergílio FS, Silva LL, Cruz GA. Síndrome de Gardner: relato de caso e breve revisão da literatura. Medicina (Ribeirão Preto, Online.). 2017; 50(5):326-32.

22. Toukacz DE, Silva MA, Salmeron S, Gottardo VD, Figueira Júnior HC. Osteoma central mandibular: relato de caso clínico. Rev UNINGÁ. 2019; 56(S7):202-14.

23. Moura LB, Ballardin L, Soldati DC, Etges A, Torriani MA, Chagas Júnior OL. Osteoma periférico mandibular - relato de caso. Full Dent. Sci. 2016;7(26):47-52.

24. Gundewar S, Kothari DS, Mokal NJ, Ghalme M. Osteomas da região craniofacial: uma série de casos e revisão da literatura. Indian J Plast Surg. 2013;46(3):479-85.

\section{CONFLITO DE INTERESSES}

Os autores declaram não haver conflitos de interesse

AUTOR PARA CORRESPONDÊNCIA

\section{Vivian Bentes de Oliveira \\ Escola Superior de Ciências do Amazonas Universidade do Estado do Amazonas, ESA-UEA 69050-030 Manaus - AM, Brasil \\ E-mail: vbo.odo16@uea.edu.br}

